\title{
The Behavior Rating Inventory of Executive Function and Continuous Performance Test in Preschoolers with Attention Deficit Hyperactivity Disorder
}

\author{
H. Tuna Çak ${ }^{1}$, S. Ebru Çengel Kültür ${ }^{1}$, Bahar Gökler ${ }^{1}$, Ferhunde Öktem ${ }^{1}$, and Candan Taşkıran² \\ ${ }^{1}$ Department of Child and Adolescent Psychiatry, Hacettepe University Faculty of Medicine, Ankara, Turkey \\ ${ }^{2}$ Department of Child and Adolescent Psychiatry, Dr. Sami Ulus Maternity and Children's Health and Diseases Training and Research Hospital, \\ Ankara, Turkey
}

Objective The aim of this study is to examine performance-based measures and behavioral ratings of executive functions (EF) as a component of preschool attention deficit hyperactivity disorder (ADHD).

Methods Twenty-one 4-to-6-year-old children with ADHD and 52 children with no psychopathology, matched on age, gender, socioeconomic status, and parental education, were enrolled. Parents were interviewed with the use of The Schedule for Affective Disorders and Schizophrenia for School-Age Children Present and Lifetime version. The Conners' Kiddie Continuous Performance Test (K-CPT) was administered to the children, and the Behavior Rating Inventory of Executive Function-Preschool version (BRIEF-P) and the Conners' Parent Rating Scale-Revised/Short Form (CPRS-R/S) were filled out by the parents.

Results All BRIEF-P and CPRS-R/S scores, the K-CPT measures of inattention and impulsivity were higher in the ADHD group. The CPRS-R/S ADHD index was strongly correlated with inhibition and related indexes in the BRIEF-P and was moderately correlated with inattention measures in the K-CPT.

Conclusion The current study is one of the few to investigate the features of preschool ADHD with the use of behavioral ratings of EF and a performance-based measure. Our results suggest that the BRIEF-P was able to identify behavioral difficulties in inhibition and working memory and that the K-CPT identified difficulties indicating inattention. The findings of this study support the use of a combination of methods for a complete evaluation of preschoolers with inattentive and hyperactive/impulsive behavior, the application of rating scales for screening ADHD symptoms, and the measurement of behavioral correlates of EF, along with performance-based measures.

Psychiatry Investig 2017;14(3):260-270

Key Words Preschool, Attention deficit hyperactivity disorder, Conners' Kiddie Continuous Performance Test, Behavior Rating Inventory of Executive Function-Preschool version.

\section{INTRODUCTION}

Attention deficit hyperactivity disorder (ADHD) is a common impairing neuropsychiatric disorder. ${ }^{1}$ Children with ADHD show developmentally inappropriate levels of inattentive and/or hyperactive impulsive behaviors in multiple settings. ADHD is known to emerge during the preschool

Received: April 23, 2016 Revised: June 18, 2016

Accepted: July 30, 2016 Available online: February 15, 2017

$\bowtie$ Correspondence: H. Tuna Cak, MD

Department of Child and Adolescent Psychiatry, Hacettepe University Faculty of Medicine, Sihhiye, Ankara 06100, Turkey

Tel: +90 312 3051150, Fax: +90 312 3051151, E-mail: tunacak@yahoo.com

(c) This is an Open Access article distributed under the terms of the Creative Commons Attribution Non-Commercial License (http://creativecommons.org/licenses/by$\mathrm{nc} / 4.0$ ) which permits unrestricted non-commercial use, distribution, and reproduction in any medium, provided the original work is properly cited. years. ${ }^{2}$ Recent meta-analytic reviews have reported that nearly $10 \%$ of preschoolers meet the full diagnostic criteria for ADHD. ${ }^{3}$ Accordingly, the number of preschool children presenting to clinicians for assessment of attention problems is growing, and diagnosing ADHD in preschool years brings potential benefits of early identification and intervention. ${ }^{4}$

ADHD is diagnosed from careful clinical evaluation. Although not diagnostic, rating scales, checklists, and neuropsychological tests may be helpful in providing evidence of the disorder. Neuropsychological impairment in the areas of sustained attention, inhibitory control, working memory (WM), and other areas of executive functions (EF) has been consistently shown to be present in school-age children with ADHD. ${ }^{5}$ Despite the finding that approximately $10 \%$ of preschoolers meet the full diagnostic criteria for ADHD, this 
disorder has been studied less extensively in preschoolers than in their school-age counterparts. Furthermore, in contrast to assessment methods in school-age children, the availability of reliable and valid measures of neuropsychological impairment in preschool children is much more limited.

Various continuous performance tests (CPT) are used, both in research and practice, to assess sustained attention, inhibitory control, and consistency of response. ${ }^{6-9}$ The Conners' Kiddie Continuous Performance Test (K-CPT) is one of the versions of $\mathrm{CPT}$ with minimal language and memory demands, created for use in younger children..$^{10}$ However, few validity studies have used the K-CPT in preschool ADHD.

There are mainly two kinds of rating scales for teachers and parents that are useful in the evaluation of ADHD symptoms: those that directly address ADHD symptoms and those that focus on EF behaviors. ADHD symptoms have been suggested to arise from a general executive dysfunction (ED). However, ED is related to various presentations, and there is a need to understand the executive domains that are more specific to $\mathrm{ADHD}$. In a meta-analytic review, $\mathrm{ED}$ in the domains of response inhibition, planning, vigilance, and WM were mentioned in the neuropsychological heterogeneity of ADHD. ${ }^{11}$ These deficits in EF were suggested to emerge in the preschool period; in particular, inhibitory control deficits observed in preschoolers seemed to predict later EF problems associated with ADHD. ${ }^{12}$ Given the concerns about the ecological validity of many performance-based EF measures in the preschool years, behavioral assessments of ED were developed. The Behavior Rating Inventory of Executive Function-Preschool version (BRIEF-P) is used to assess EF in 2-to-5-year-old children as perceived by their parents or teachers. ${ }^{13}$ Its usefulness in identifying disruptive behavior disorders has been evaluated in a number of studies. ${ }^{13-16}$ However, there is scarce literature regarding preschoolers with $\mathrm{ADHD}$ with respect to $\mathrm{ED}$ and their behavioral associations. ${ }^{9,12,17,18,19}$ Valid and reliable assessment tools to improve the differentiation of specific impairments of ADHD early in life can be valuable for the planning of interventions in affected children.

Considering the above-summarized knowledge, the aim of this study is to examine possible impairment in performancebased measures of attention and impulsivity and behavioral ratings of $\mathrm{EF}$ as a component of preschool ADHD. In addition, we aim to examine the relation between parent ratings of ADHD symptoms, performance-based measures of attention and impulsivity, and behavioral ratings of EF.

\section{METHODS}

\section{Participants}

Twenty-five children, ages 4 to 5 years old, admitted to the
Hacettepe University Child and Adolescent Psychiatry Outpatient Clinic and diagnosed with ADHD by an experienced child and adolescent psychiatrist according to the criteria in the Diagnostic and Statistical Manual of Mental Disorders, fourth edition (DSM-IV), were enrolled in the study group. For the control group, a total of 60 children with no identified psychopathology were selected from the BRIEF-P standardization sample (Gokler, Oktem \& CengelKultur, 2009) and were matched individually to each child in the ADHD group on age, gender, socioeconomic status (SES), and parental education. Children with any kind of chronic/neurologic disease, mental retardation, developmental language disorder, or autistic spectrum disorder; those attending any kind of special education program; those who could not complete the psychiatric assessments required; and those with a total intelligence quotient (IQ) below 80 in the Stanford-Binet V Intelligence Scale (SB-V) were excluded from the study. None of the children had received any psychopharmacologic treatment or intervention for any psychiatric disorder at the time of assessment.

\section{Measures}

A sociodemographic data form developed by the research group was used to obtained sociodemographic information on the participants.

The Schedule for Affective Disorders and Schizophrenia for School-Age Children-Present and Lifetime version (KSADS-PL) is a semi-structured diagnostic interview designed to assess current and past episodes of psychiatric disorders according to the DSM-IV. ${ }^{20}$ The K-SADS-PL was not originally designed for preschool children. However, Birmaher et al. carried out a psychometric study to assess the reliability of the K-SADS-PL in preschool children ages 2 to 5 years, suggesting that it was a reliable instrument for evaluating psychiatric disorders in preschoolers, particularly ADHD, oppositional defiant disorder (ODD), and anxiety, mood, and elimination disorders. ${ }^{21}$ The standardization of the instrument for the Turkish population has been completed in 2004. ${ }^{22}$

The Conners' Parent Rating Scale-Revised/Short Form (CPRS-R/S) is a widely used instrument for screening and evaluating ADHD-related symptoms in children and adolescents ages 3 to 17 years with the use of parent and teacher rating forms. ${ }^{23}$ The CPRS-R/S contains 27 items that are rated on a four-point scale and covers a set of the scales on items such as oppositional behavior, cognitive problems/inattention, hyperactivity, and ADHD index. The adaptation and standardization for Turkish children was done by Kaner et al. ${ }^{24}$

The Conners' Kiddie Continuous Performance Test version 5 (K-CPT V5) is a computer-based program for assessing attention problems in children 4 and 5 years old. ${ }^{10}$ The admin- 
istration of the test takes about 7.5 minutes; the stimuli consist of a series of pictures of objects that are familiar to preschool children, such as a boat or a ball. The stimulus intervals are either 1.5 or 3 seconds, and the children are asked to press the space bar or click the mouse for every picture that appears on the screen, except the ball. For each interstimulus interval, 5 blocks consisting of 20 pictures are presented. The K-CPT $\mathrm{V} 5$ reports the results on omission and commission rates, overall hit reaction time (Hit RT), overall hit reaction time standard error (Hit RT SE), variability, detectability (d'), response style $(\beta)$, perseverations, hit reaction time by block change (Hit RT Block Change), hit reaction time standard error by block change (Hit SE Block Change), hit reaction time by interstimulus interval change (Hit RT ISI Change), and hit reaction time standard error by interstimulus interval change (Hit SE ISI Change). The K-CPT measures are grouped into indicators of inattention, impulsivity, and vigilance.

The Behavior Rating Inventory of Executive Function-Preschool version (BRIEF-P) consists of 63 items that measure various behavioral manifestations of EF based on parent or teacher ratings, within the context of the child's everyday environment, in children ages 2 to 5 years old. ${ }^{25}$ The BRIEF-P covers the scales inhibit (I), shift (S), emotional control (EC), working memory (WM), and plan/organize (PO). These scales form three broad indexes: inhibitory self-control (ISC), flexibility (F), and emergent metacognition (EM), as well as one composite score, the global executive composite (GEC). The standardization of the instrument for the Turkish population has been completed in $2009 .{ }^{19}$

The Stanford-Binet V Intelligence Scale (SB-V) is an individually administered assessment of intelligence and cognitive abilities in children and adults. ${ }^{26}$ This instrument provides scores for full-scale, verbal, and nonverbal IQ, as well as five composite scores in the areas assessed. The standardization of the instrument for the Turkish population has been completed in $2009 .^{19}$

\section{Procedure}

A clinical psychologist administeredthe SB-V. Two children from the study group and 5 children from the control group with total IQs below 80 were excluded from the study. The remaining children were subjected to further evaluation on a different day to avoid the possibility of different carryover effects between the ADHD and control groups. Parents were interviewed with the use of the K-SADS-PL by a child and adolescent psychiatrist other than the one who carried out the first evaluation to confirm the presence or absence of ADHD diagnosis and other psychiatric disorders. A sociodemographic data form was completed by the same psychiatrist during the parent interview. The children were allowed to spend time in a playroom and were interviewed after their parents by the same psychiatrist. Stuttering and somatoform disorders were questioned additionally. Parents were asked to fill out the BRIEF-P and the CPRS-R/S. The K-CPT was administered to the children in a quiet room. The short practice test included in the software was used before the administration of the full test to make sure the instructions were fully understood. A one-warning approach was used in cases in which the child deviated from the task; if the deviation continued, no further prompts were given. Two children from the ADHD group and 3 children from the control group were excluded from the study for not being able follow through in the K-CPT. Institutional review and approval were obtained from the Ethics Committee. All parents gave a written informed consent, and the children agreed to participate in the study.

\section{Statistical analysis}

The software SPSS 18.0 was used for statistical analyses. Continuous variables in normal distributions were analyzed by using the Kolmogorov-Smirnov test with Lilliefors significance correction. Student's t test and chi-square tests were applied to determine the differences in continuous and categorical variables, respectively, between the ADHD and control groups. Effect size (ES) values were computed by using the d statistic. The ES is an objective and standardized measure of the magnitude of change that one variable produces in another variable as reflected in the difference between two means, independent of the sample size. ${ }^{27}$ The interpretation of the ES (d) is based on a convention suggested by Cohen (1988), such that 0.20 is considered as a "small" ES, 0.50 as "medium," and 0.80 or greater as "large." Because the BRIEF-P, CPRS-R/S, and K-CPT measures were distributed normally in the ADHD and control groups, the correlation coefficients and their significance were calculated by using the Pearson test with scatterplots. BRIEF-P subscale scores and the K-CPT results in the two groups were investigated using repeated measures analysis of variance. Greenhouse-Geisser correction was used when the sphericity assumption was violated. All tests were two-tailed, and a 5\% type I error was used to infer statistical significance.

\section{RESULTS}

By design, the ADHD and control samples were not significantly different in age, gender, SES, and parental education. In addition, other factors, such as parental age, maternal occupation status, number of siblings, prematurity, perinatal complications, major developmental milestones, and total IQ scores, did not significantly differ between the ADHD and 
Table 1. Sociodemographic features of the ADHD and control groups

\begin{tabular}{|c|c|c|c|}
\hline & $\operatorname{ADHD}(\mathrm{N}=21)$ & CONTROL $(\mathrm{N}=52)$ & Statistics \\
\hline Age (months) (mean \pm SD) & $58.1 \pm 8.3$ & $56.9 \pm 9.1$ & $\mathrm{t}=0.95 \mathrm{p}=0.348$ \\
\hline Male/Female $(\mathrm{N})$ & $18 / 3$ & $34 / 18$ & $\chi^{2}=3.02 \mathrm{p}=0.082$ \\
\hline Maternal age (years) $($ mean $\pm S D)$ & $31.9 \pm 5.7$ & $35.4 \pm 5.7$ & $\mathrm{t}=1.62 \mathrm{p}=0.110$ \\
\hline Paternal age (years) (mean $\pm S D)$ & $36.9 \pm 5.0$ & $40.2 \pm 4.6$ & $\mathrm{t}=1.72 \mathrm{p}=0.060$ \\
\hline Maternal education (years) (mean $\pm \mathrm{SD})$ & $10.3 \pm 4.2$ & $11.4 \pm 3.0$ & $\mathrm{t}=0.95 \mathrm{p}=0.350$ \\
\hline Paternal education (years) (mean \pm SD) & $10.4 \pm 3.8$ & $11.4 \pm 4.1$ & $\mathrm{t}=0.78 \mathrm{p}=0.440$ \\
\hline Working mothers $(\mathrm{N})$ & 11 & 30 & $\chi^{2}=1.12 \mathrm{p}=0.289$ \\
\hline Number of siblings (median, min-max) & $0(0-1)$ & $1(0-3)$ & $\mathrm{Z}=1.62 \mathrm{p}=0.110$ \\
\hline Premature birth history (N) & 3 & 14 & $\chi^{2}=2.25 \mathrm{p}=0.325$ \\
\hline Perinatal complication history $(\mathrm{N})$ & 2 & 4 & $\chi^{2}=3.19 \mathrm{p}=0.202$ \\
\hline \multicolumn{4}{|c|}{ Developmental milestones (months) (mean \pm SD) } \\
\hline Walking & $12.6 \pm 3.2$ & $12.8 \pm 4.1$ & $\mathrm{t}=0.79 \mathrm{p}=0.430$ \\
\hline Talking & $20.5 \pm 3.8$ & $18.9 \pm 4.4$ & $\mathrm{t}=0.16 \mathrm{p}=0.145$ \\
\hline SB-V total IQ (mean \pm SD) & $98.13 \pm 19.45$ & $109.54 \pm 33.79$ & $\mathrm{t}=1.178 \mathrm{p}=0.247$ \\
\hline
\end{tabular}

ADHD: attention deficit hyperactivity disorder, SB-V: Stanford-Binet V Intelligence Scale, IQ: intelligence quotient, p: p-vaue, t: Student's variable, $\mathrm{Z}$ : $\mathrm{Z}$ score, $\chi^{2}$ : chi-square distribution

control groups (Table 1). The comorbidity rate in the ADHD group was $76 \%(\mathrm{n}=16)$. The most common comorbid psychiatric disorders were oppositional defiant disorder $(n=9$, $43 \%)$, nocturnal enuresis $(n=5,24 \%)$, and separation anxiety disorder $(n=3,14 \%)$.

The BRIEF-P raw scores were converted into $\mathrm{T}$ scores by using appropriate age and gender norms. All BRIEF-P scales/ indexes were significantly higher in the ADHD group, with large ES values ranging between 2.89 and 4.66. The biggest ES values in the clinical scales and indexes were observed in I, WM, EM, and GEC. All CPRS-R/S scales were significantly higher in the ADHD group, with large ES values ranging between 1.03 and 1.58. The omission and commission rates, Hit RT SE, variability, Hit RT ISI change, and Hit RT SE ISI change were significantly higher in the ADHD group, with medium-to-large ES values ranging between 0.72 and 0.95 in the K-CPT measures. The biggest ES values were observed in the changes in Hit RT ISI and Hit RT SE ISI (Table 2). MANOVA results revealed an overall group effect for the five BRIEF$P$ subscales $\left(F=7.765, p<0.001\right.$, Pillai's Trace $\left.=0.32 ; \eta^{2}=0.25\right)$ and K-CPT scores $(\mathrm{F}=3.918, \mathrm{p}=0.003$, Pillai's Trace $=0.34$; $\left.\eta^{2}=0.75\right)$. Group differences across BRIEF-P subscales and K$\mathrm{CPT}$ scores were further explored in a profile analysis (Figure 1). A significant withingroups main effect was found, showing different scale elevations across the BRIEF-P subscales $\left(\mathrm{F}=5.81, \mathrm{p}<0.001, \eta^{2}=0.08\right)$ and the K-CPT scores $(\mathrm{F}=3.92$, $\left.\mathrm{p}=0.003, \eta^{2}=0.75\right)$. Children in the ADHD group scored significantly higher than typically developing controls in all of the five BRIEF-P EF domains.

When all the measures were compared on gender within the ADHD and control groups separately, only the CPRS-R/S hyperactivity scale score was significantly higher for boys in the ADHD group ( $\mathrm{t}=4.24, \mathrm{p}=0.001)$. There were no significant differences in scores in any scale or measure regarding the presence of psychiatric comorbidity in the ADHD group.

All CPRS-R/S scales were positively correlated with the BRIEF-P scales/indexes for the whole sample, with moderate-to-strong correlation coefficients ranging from 0.49 to 0.79 (Figure 2). When the analysis was replicated for the ADHD group only, the CPRS-R/S oppositional scale was moderately correlated with the BRIEF-P I scale and the ISC and GEC indexes. The CPRS-R/S cognitive problems/inattention scale was not correlated with any of the BRIEF-P scales/indexes. The CPRS-R/S hyperactivity scale was moderately correlated with the BRIEF-P I scale and ISC index. The CPRS-R/S ADHD index was strongly correlated with the BRIEF-P I scale and ISC index and was moderately correlated with the GEC index (Figure 3).

The K-CPT measures were analyzed for correlations between ADHD symptoms in the CPRS-R/S. For the whole sample, the CPRS-R/S oppositional scale was correlated with omission rate, Hit RT SE, variability, and Hit RT ISI change. The CPRS-R/S cognitive problems/inattention scale was correlated with omission rate. The CPRS-R/S hyperactivity scale, similarly to the oppositional scale, was correlated with omission rate, Hit RT SE, variability, and Hit RT ISI change plus perseverations. The CPRS-R/S ADHD index, similarly to the oppositional scale, was correlated with omission rate, Hit RT SE, variability, and Hit RT ISI change, with higher correlation coefficients indicating a moderate relationship with the 
Table 2. BRIEF-P indexes, CPRS-R/S scales, and K-CPT measures in the ADHD and control groups

\begin{tabular}{|c|c|c|c|c|}
\hline & $\mathrm{ADHD}($ mean $\pm \mathrm{SD})$ & CONTROL $($ mean \pm SD) & Statistics & $\mathrm{d}$ \\
\hline \multicolumn{5}{|l|}{ BRIEF-P scales/indexes } \\
\hline Inhibit & $96.15 \pm 11.12$ & $55.04 \pm 11.72$ & $\mathrm{t}=11.79 \mathrm{p}<0.001$ & 3.59 \\
\hline Shift & $89.45 \pm 12.24$ & $51.65 \pm 9.97$ & $\mathrm{t}=10.99 \mathrm{p}<0.001$ & 3.38 \\
\hline Emotional control & $86.23 \pm 12.26$ & $52.72 \pm 10.88$ & $\mathrm{t}=9.49 \mathrm{p}<0.001$ & 2.89 \\
\hline Working memory & $101.12 \pm 12.95$ & $53.83 \pm 10.91$ & $\mathrm{t}=12.99 \mathrm{p}<0.001$ & 3.95 \\
\hline Plan/Organize & $91.85 \pm 10.49$ & $54.13 \pm 10.91$ & $\mathrm{t}=11.51 \mathrm{p}<0.001$ & 3.52 \\
\hline Inhibitory self-control & $95.91 \pm 11.67$ & $54.61 \pm 11.44$ & $\mathrm{t}=12.12 \mathrm{p}<0.001$ & 3.57 \\
\hline Flexibility & $92.65 \pm 12.75$ & $52.39 \pm 10.63$ & $\mathrm{t}=11.26 \mathrm{p}<0.001$ & 3.43 \\
\hline Emergent metacognition & $100.46 \pm 11.61$ & $54.09 \pm 9.54$ & $\mathrm{t}=13.58 \mathrm{p}<0.001$ & 4.36 \\
\hline Global executive composite & $103.05 \pm 10.07$ & $54.39 \pm 10.79$ & $\mathrm{t}=15.21 \mathrm{p}<0.001$ & 4.66 \\
\hline \multicolumn{5}{|l|}{ CPRS-R/S scales } \\
\hline Oppositional & $11.32 \pm 3.38$ & $6.60 \pm 3.94$ & $\mathrm{t}=4.01 \mathrm{p}<0.001$ & 1.29 \\
\hline Cognitive problems/Inattention & $10.12 \pm 3.61$ & $4.65 \pm 5.66$ & $\mathrm{t}=3.29 \mathrm{p}=0.002$ & 1.15 \\
\hline Hyperactivity & $10.11 \pm 3.98$ & $5.35 \pm 5.17$ & $\mathrm{t}=3.11 \mathrm{p}=0.004$ & 1.03 \\
\hline ADHD index & $20.41 \pm 6.40$ & $8.13 \pm 8.92$ & $\mathrm{t}=4.51 \mathrm{p}<0.001$ & 1.58 \\
\hline \multicolumn{5}{|l|}{ K-CPT measures } \\
\hline Omissions (\%) & $53.85 \pm 9.52$ & $47.59 \pm 7.62$ & $\mathrm{t}=2.15 \mathrm{p}=0.039$ & 0.73 \\
\hline Commissions (\%) & $55.84 \pm 7.26$ & $49.44 \pm 9.90$ & $\mathrm{t}=2.17 \mathrm{p}=0.037$ & 0.74 \\
\hline Hit RT & $50.43 \pm 10.36$ & $46.57 \pm 9.73$ & $\mathrm{t}=1.14 \mathrm{p}=0.264$ & 0.38 \\
\hline Hit RT SE & $54.41 \pm 8.49$ & $47.36 \pm 9.90$ & $\mathrm{t}=2.26 \mathrm{p}=0.031$ & 0.76 \\
\hline Variability & $56.71 \pm 12.09$ & $48.52 \pm 10.45$ & $\mathrm{t}=2.15 \mathrm{p}=0.039$ & 0.72 \\
\hline Detectability (d') & $54.94 \pm 7.79$ & $48.76 \pm 10.76$ & $\mathrm{t}=1.94 \mathrm{p}=0.061$ & 0.66 \\
\hline Response Style $(\beta)$ & $47.44 \pm 13.24$ & $51.39 \pm 13.68$ & $\mathrm{t}=0.87 \mathrm{p}=0.392$ & 0.29 \\
\hline Perseverations (\%) & $52.63 \pm 12.09$ & $45.47 \pm 10.07$ & $\mathrm{t}=1.91 \mathrm{p}=0.065$ & 0.64 \\
\hline Hit RT Block change & $49.50 \pm 9.28$ & $46.63 \pm 7.55$ & $\mathrm{t}=1.01 \mathrm{p}=0.320$ & 0.34 \\
\hline Hit SE Block change & $49.62 \pm 10.41$ & $45.96 \pm 8.12$ & $\mathrm{t}=1.16 \mathrm{p}=0.253$ & 0.39 \\
\hline Hit RT ISI change & $55.39 \pm 11.70$ & $45.76 \pm 8.37$ & $\mathrm{t}=2.81 \mathrm{p}=0.008$ & 0.95 \\
\hline Hit SE ISI change & $56.35 \pm 7.99$ & $47.70 \pm 12.52$ & $\mathrm{t}=2.42 \mathrm{p}=0.021$ & 0.82 \\
\hline
\end{tabular}

Effect size $\mathrm{d}=($ mean of ADHD group-mean of control group)/pooled SD of the two groups. ADHD: attention deficit hyperactivity disorder, BRIEF-P: Behavior Rating Inventory of Executive Function-Preschool version, CPRS-R/S: Conners' Parent Rating Scale-Revised/Short Form, K-CPT: Kiddie Continuous Performance Test, SD: standard deviation, RT: reaction time, SE: standard error, ISI: interstimulus interval, p: pvaue, $t$ : Student's variable, Effect size $\mathrm{d}=$ (mean of ADHD group-mean of control group)/pooled SD of the two groups

omission rate and Hit RT ISI change (Figure 2). When analyzed for the ADHD group only, the CPRS-R/S oppositional scale was moderately correlated with variability. The CPRS-R/S cognitive problems/inattention scale was not correlated with any of the K-CPT measures. The CPRS-R/S hyperactivity scale was moderately correlated with variability and response style. The CPRS-R/S ADHD index had the most significant correlations with the K-CPT measures, namely on omission rate, Hit RT SE, variability, detectability, response style, Hit RT ISI change, and Hit SE ISI change, with a strong correlation coefficient on the variability measure (Figure 3 ).

\section{DISCUSSION}

Consistent with the idea that ADHD symptoms emerge during the preschool years, our sample of preschool children with ADHD showed significantly higher scores in parentrated behavioral scales of ADHD in the CPRS-R/S and in all the scales and indexes of the BRIEF-P, which measure parent-rated behavioral correlates of EF. Children with ADHD also showed significantly lower performance in the K-CPT measures related to inattention (omission and commission rates, Hit RT SE, variability, Hit RT ISI change, and Hit SE ISI change) and impulsivity (commission rate). The CPRS-R/S ADHD index was strongly correlated with inhibition and re- 


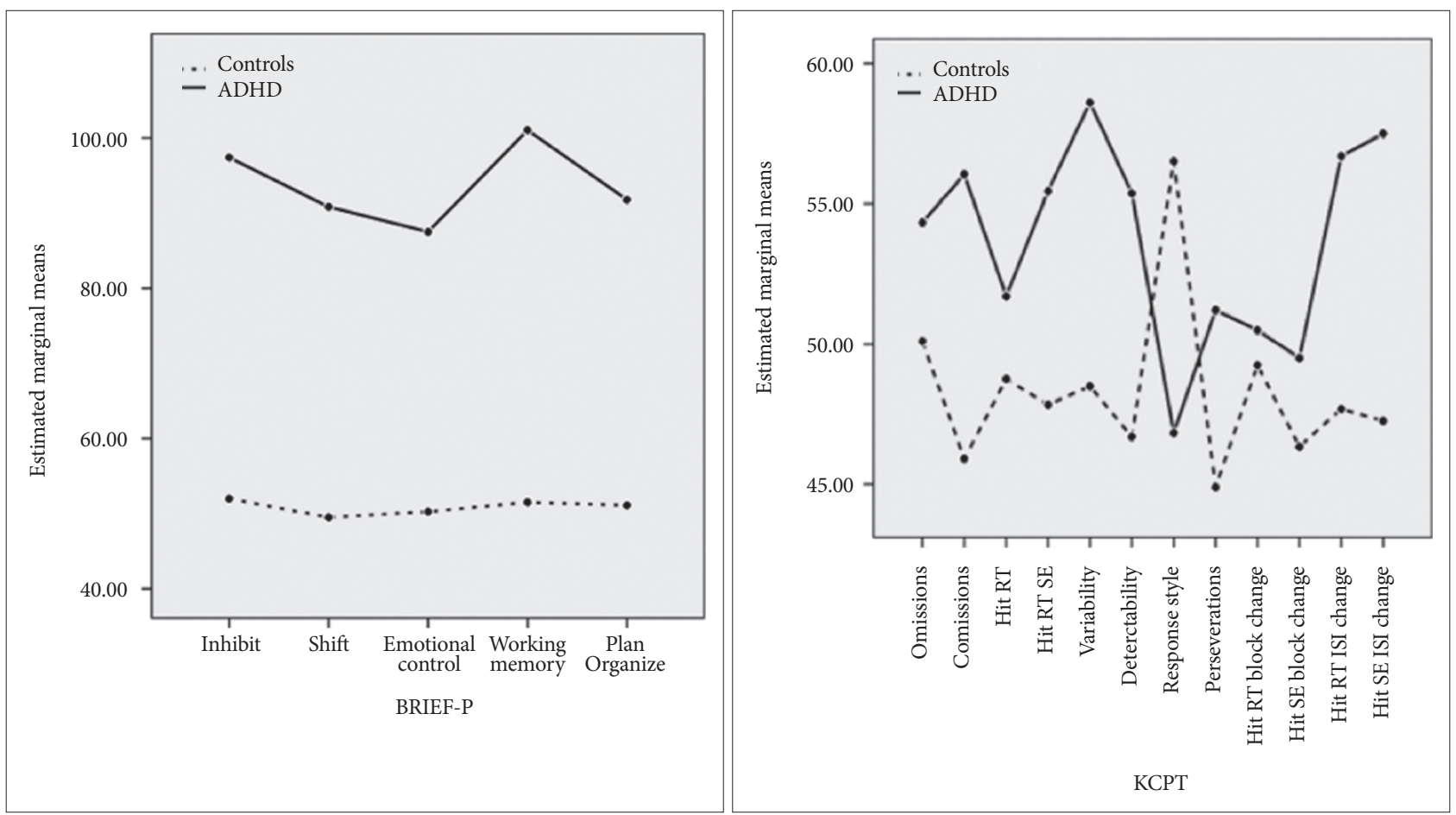

Figure 1. Profiles for ADHD and the Control Groups across BRIEF-P scales and K-CPT scores. ADHD: attention deficit hyperactivity disorder, BRIEF-P: Behaviour Rating Inventory of Executive Function-Preschool version, K-CPT: Kiddie Continuous Performance Test, RT: reaction time, SE: standard error, ISI: interstimulus interval.

lated indexes in the BRIEF-P and was moderately correlated with inattention measures in the $\mathrm{K}-\mathrm{CPT}$ in the ADHD group.

The ADHD and control groups were not significantly different in age, gender, SES, parental education, parental age, maternal occupation status, number of siblings, prematurity, perinatal complications, major developmental milestones, and total IQ scores. The similarities made the control group more comparable in the ADHD-related domains, reducing the confounding effects of demographic features and IQ. No significant differences were found regarding the presence of psychiatric comorbidity in the ADHD group. Similarly Ezpeleta and Granero showed similar executive difficulties in preschoolers with $\mathrm{ADHD}$ and ODD comorbid ADHD using the BRIEF-P and the K-CPT and suggested that, EF deficits assessed with a performance-based measure or with behavioral descriptions are specific to preschoolers with ADHD, in comparison with those with ODD. ${ }^{28}$

When considered in the scales and measures used, the ES mostly exceeded 3 in the BRIEF-P scales and indexes, was close to 1 in the CPRS-R/S scales, and was around 0.75 in the statistically significant K-CPT measures. The ES can be interpreted in terms of the percent of nonoverlap of scores between the study group and the control group. ${ }^{25}$ An ES of 0.0 indicates that the distribution of scores for the study group overlaps completely with that for the control group; that is, there is $0 \%$ nonoverlap. ${ }^{27} \mathrm{An} \mathrm{ES}$ of 0.7 , similarly to our K-CPT mea- sures, indicates a nonoverlap of $43 \%$; an ES of 1 , similarly to our CPRS-R/S scales, indicates a nonoverlap of $58.9 \%$; and an ES of 2, similarly to our BRIEF-P scales/indexes, indicates a nonoverlap of $81.1 \%$ between the two distributions. ${ }^{27}$ The results of our study reveal a small overlap in the BRIEF-P scale/index distributions between the preschool ADHD and control groups, which has a high practical significance. In one of the few studies that examined the validity of the BRIEF$\mathrm{P}$ in preschool children with $\mathrm{ADHD}$, the ES values in the BRIEF-P scales/indexes ranged from 0.8 to 2.4 , which are smaller than the ES values in our study. ${ }^{12}$ Although the mean ages of the samples in that study and in the present research are similar, the age range is wider; further, children 36 to 71 months old were included in the former study. The small sample sizes in both studies might have increased the effect in younger children and contributed to the difference in ES between the two studies. On the other hand, similarly to our findings, the largest ES values in the BRIEF-P were observed on the I, WM, EM, and GEC in the study by Mahone and Hoffmann. ${ }^{12}$ Again, similarly to our findings, the I and WM scales in the BRIEF-P classified the sample into ADHD and control groups with a desirable accuracy of $86 \%$ in a recent study. ${ }^{16}$ Among preschoolers with ADHD, inhibition deficits are more pronounced and seem to predict later EF problems associated with ADHD. ${ }^{12,29}$ The findings regarding the role of WM as measured by performance-based tests have thus far 


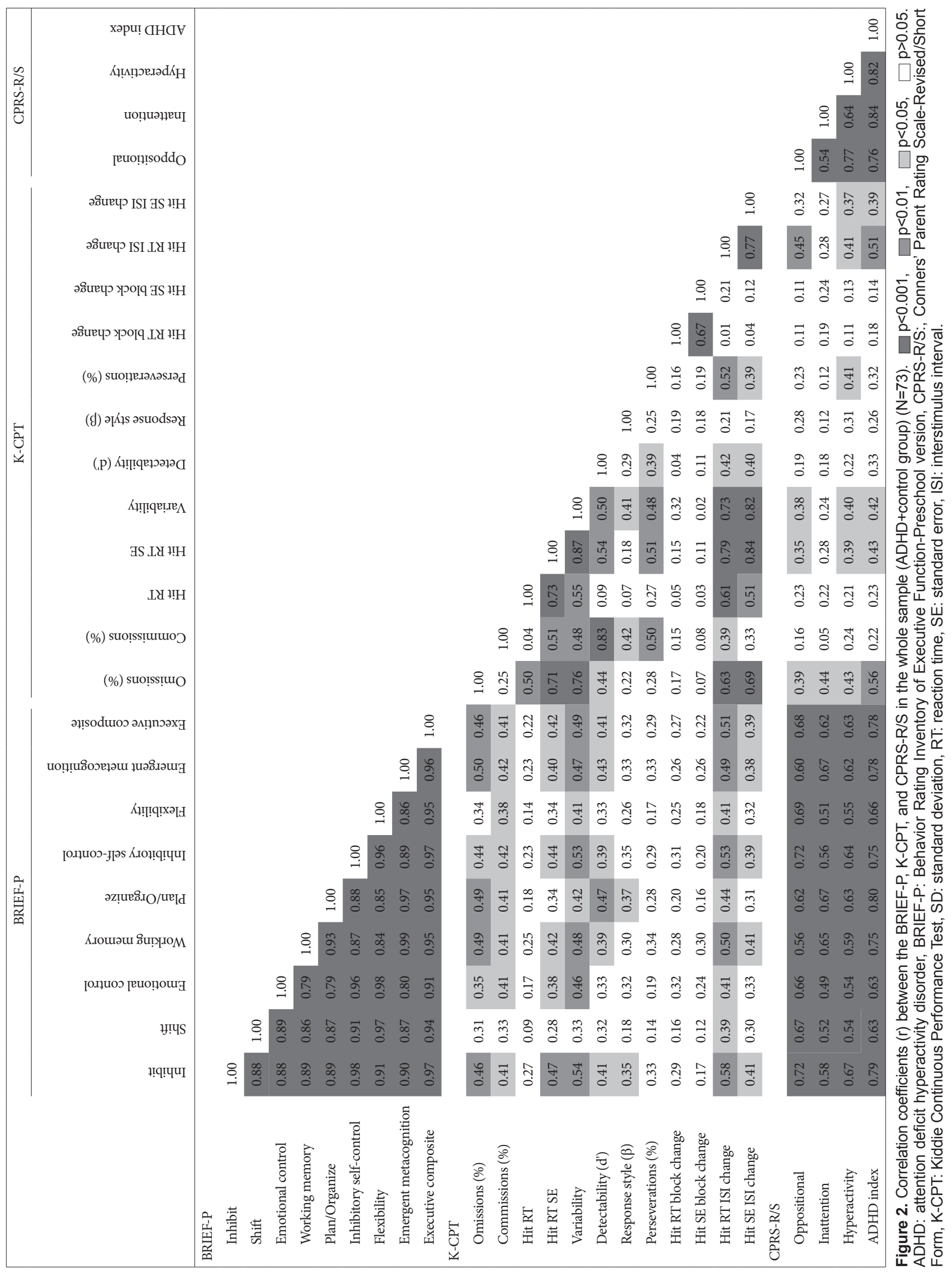




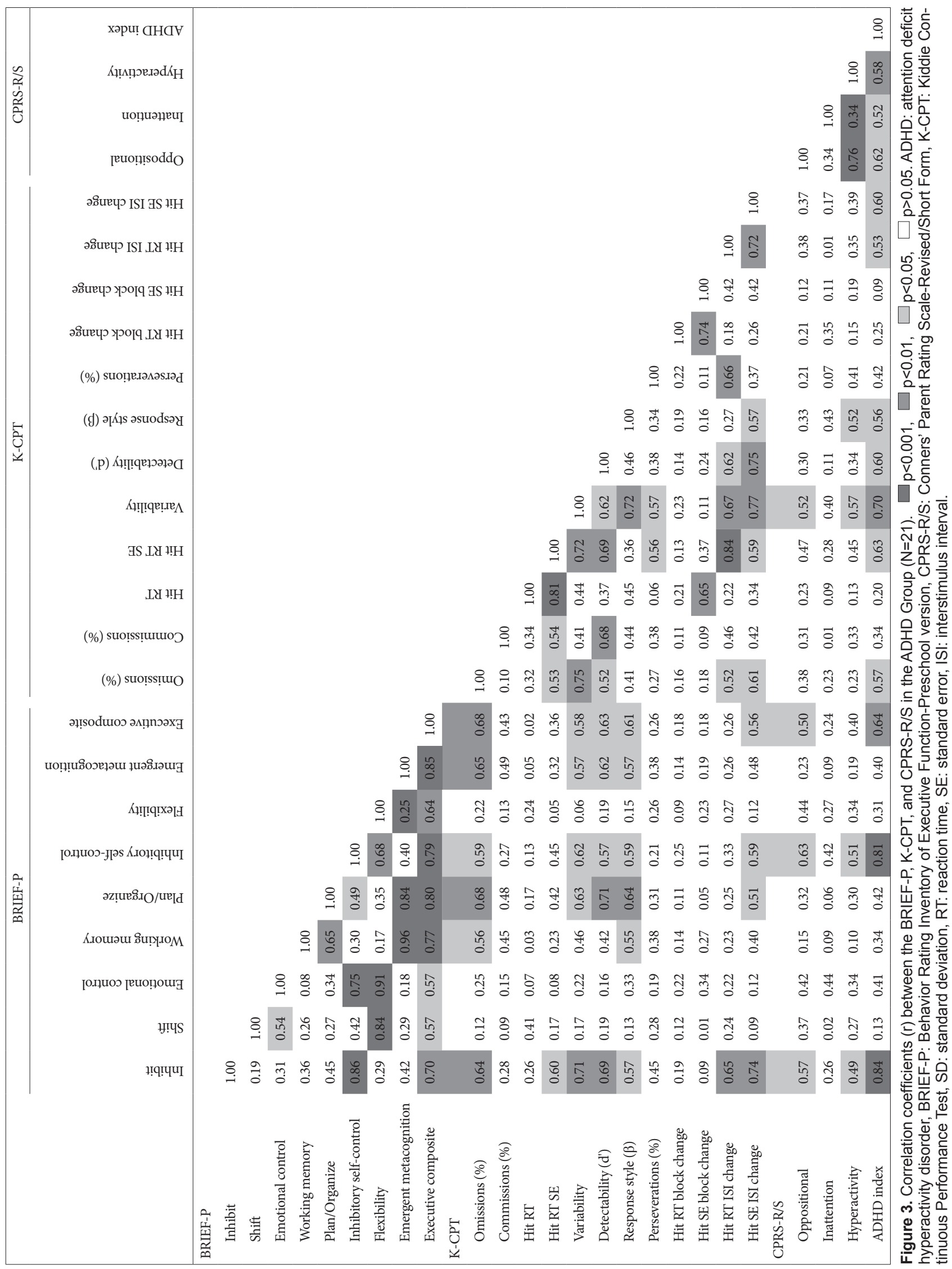


been less consistent. ${ }^{30-33}$ The present study reports evidence for a link between parents' behavioral ratings of WM deficits and increased levels of ADHD symptoms. It should be noted that this finding does not establish strong evidence to show clear WM deficits in preschool ADHD and that WM was not evaluated through performance-based measures. Longitudinal research with larger samples is needed to clarify the role of WM in the early development of ADHD. Nevertheless, the present findings support the clinical utility of the BRIEF-P in differentiating between children with ADHD and typically developing controls.

The K-CPT has been shown to have adequate reliability in children 4 and 5 years old, differentiating between subjects with and without ADHD based on a sample of 454 children. $^{10}$ In the present study, the omission rate, Hit RT SE, variability, and Hit RT ISI change as indicators of inattentiveness, the commission rate as an indicator of impulsivity, and the Hit SE ISI change as an indicator of vigilance were significantly higher in the ADHD group. Similarly to our results, several studies have reported higher commission and omission rates in preschool children with ADHD; further, preschool children with hyperactive and oppositional behaviors have been observed to have more omissions, faster Hit RT SE, and greater variability. ${ }^{9,34-38}$ In addition, Barnard et al..$^{39}$ suggested that reaction time and omission rate were associated with parent-reported symptoms of EF deficits in preschool children. Despite these specific results congruent with the previous literature, the ES for the significant K-CPT measures remained below 1, much lower than the ES calculated for the BRIEF-P. Correspondingly, the predictive power of the K$\mathrm{CPT}$ is said to be moderate, with ES values ranging between 0.58 and 1.0 in the above-mentioned comparable study. ${ }^{9}$ The much lower ES in the K-CPT than that in the BRIEF-P can be regarded as evidence that the BRIEF-P measures different and more extensive constructs of $\mathrm{EF}$ than those assessed by the K-CPT in preschool children with ADHD.

That all CPRS-R/S scales were positively correlated with the BRIEF-P scales/indexes in the whole sample may suggest that the BRIEF-P measures parent-reported behavioral constructs of the EF that may underlie parent-rated symptoms of ADHD. In the ADHD group, symptoms of oppositional and hyperactive behavior were moderately correlated with the BRIEF-P inhibition-related scales/indexes, supporting the idea that among EF constructs, inhibition deficits are predominant in preschool ADHD. ${ }^{12,29}$ Symptoms of inattention were not correlated with any of the BRIEF-P scales/indexes. Parents of preschool children might not be able recognize symptoms of inattention because such children have not yet encountered high cognitive demand tasks. On the contrary, parent-rated ADHD symptoms were more correlated with measures of inattention, not impulsivity or vigilance, in the K-CPT. Similarly, in the study by Ezpeleta and Granero, ${ }^{28}$ the inattention measures in the K-CPT were the best in differentiating preschool children with externalizing problems from typically developing controls. Espy et $\mathrm{al}^{40}$ suggested that the BRIEF-P should be used as a broad indicator of problem behavior and not as a substitute for assessing performance-based executive skills. Therefore, we suggest the use of a combination of methods for a complete evaluation of preschoolers with inattentive and hyperactive/impulsive behavior, the application of rating scales for screening ADHD symptoms, and the measurement of behavioral correlates of $\mathrm{EF}$, along with performance-based measures. In fact, our results suggest that the BRIEF-P was able to identify gross behavioral difficulties in inhibition and that the K-CPT identified difficulties related to omission and reaction time indicating inattention. Obtaining different aspects of information in the evaluation may be helpful in planning interventions.

The strengths of the current study include the use of a DSMIV-based, multi-method (interview and rating scale) diagnostic procedure for $\mathrm{ADHD}$ and careful matching of the children in the ADHD group on age, gender, SES, and parental education. The important demographic features and total IQ scores were not significantly different between the ADHD and control groups, making the groups more homogenous and reducing possible controllable confounding effects. Nevertheless, this study also has several limitations. Although the groups were matched on several sociodemographic variables, there could be other uncontrollable variables that influenced the performance of the children in the K-CPT or the parent responses in the rating scales. It should be noted that there was only one performance-based test used (K-CPT). Further, the performance-based measures and the scales compared ADHD and typically developing children; we did not include other common disorders in the preschool population, such as ODD and anxiety disorders, and did not compare ADHD with these other disorders. The narrow age range also limits the generalization of findings to the youngest preschoolers. Because only parent ratings were included in this study, our findings may not apply to the use of the BRIEF-P and CPRS$\mathrm{R} / \mathrm{S}$ by teachers. Due to the small sample size, the number of significant correlations may also be somewhat reduced. On the other hand, although the scores analyzed were normally distributed, extreme values could have distorted the correlation coefficients. We tried to determine the effects of extreme values by using scatterplots in each correlation analysis. We did not detect such extreme values; however, the effect of even minor extreme values increases with a smaller sample size.

In spite of its limitations, the current study is one of the few to investigate the features of preschool ADHD with the use of 
behavioral ratings of EF and a performance-based measure. Despite the relatively small sample size, the ES values were consistently large, with highly significant results. Our findings support the use of the BRIEF-P in the identification and description of EF difficulties in preschool ADHD. The comparison of ratings within the five clinical scales of the BRIEF$\mathrm{P}$ proved useful in distinguishing ADHD from typically developing controls. ADHD-related difficulties were identified primarily in I and WM, suggesting that deficiencies within these two EF domains contribute to the development of ADHD. These relations should be further addressed in follow-up studies in children first assessed as younger preschoolers, in additional diagnostic groups, and in larger samples, along with multiple performance-based tests of EF. The identification of deviancies within specific areas of everyday EF at an early point in development may be useful in planning targeted interventions for use in young children with ADHD.

\section{Acknowledgments}

This project was financially supported by The Scientific and Technological Research Council of Turkey (TÜBITAK, Project ID 107S437). The sponsor of the study had no role in study design, data gathering, data analysis and data interpretation or writing the report.

\section{REFERENCES}

1. American Psychiatric Association (APA). Diagnostic and Statistical Manual of Mental Disorders, Fourth Edition. Washington, DC: American Psychiatry Association; 1994.

2. Applegate B, Lahey BB, Hart EL, Biederman J, Hynd GW, Barkley RA, et al. Validity of the age-of-onset criterion for ADHD: a report from the DSM-IV field trials. J Am Acad Child Adolesc Psychiatry 1997; 36:1211-1221.

3. Willcutt EG. The prevalence of DSM-IV attention-deficit/hyperactivity disorder: a meta-analytic review. Neurotherapeutics 2012;9:490-499.

4. Charach A, Carson P, Fox S, Ali MU, Beckett J, Lim CG. Interventions for preschool children at high risk for ADHD: a comparative effectiveness review. Pediatrics 2013;131:e1584-e1604.

5. Doyle AE. Executive functions in attention-deficit/hyperactivity disorder. J Clin Psychiatry 2006;67(Suppl 8):21-26.

6. Mahone EM, Schneider HE. Assessment of attention in preschoolers. Neuropsychol Rev 2012;22:361-383.

7. Pauli-Pott U, Becker K. Neuropsychological basic deficits in preschoolers at risk for ADHD: a meta-analysis. Clin Psychol Rev 2011;31:626637.

8. Sims DM, Lonigan CJ. Multi-method assessment of ADHD characteristics in preschool children: relations between measures. Early Child Res Q 2012;27:329-337.

9. Youngwirth SD, Harvey EA, Gates EC, Hashim RL, Friedman-Weieneth JL. Neuropsychological abilities of preschool-aged children who display hyperactivity and/or oppositional-defiant behavior problems. Child Neuropsychol 2007;13:422-443.

10. Conners CK. Conners' Kiddie Continuous Performance Test (K-CPT). Toronto: Multi-Health Systems; 2001.

11. Willcutt EG, Doyle AE, Nigg JT, Faraone SV, Pennington BF. Validity of the executive function theory of attention-deficit/hyperactivity disorder: a meta-analytic review. Biol Psychiatry 2005;57:1336-1346.

12. Mahone EM, Hoffman J. Behavior ratings of executive function among preschoolers with ADHD. Clin Neuropsychol 2007;21:569-586.

13. Gioia GA, Espy KA, Isquith PK. Behavior Rating Inventory of Execu- tive Function-Preschool Version. Lutz, FL: Psychological Assessment Resources; 2003.

14. Overgaard KR, Oerbeck B, Aase H, Torgersen S, Reichborn-Kjennerud T, Zeiner P. Emotional lability in preschoolers with symptoms of ADHD. J Atten Disord 2015 [Epub ahead of print].

15. Pritchard AE, Kalback S, McCurdy M, Capone GT. Executive functions among youth with Down Syndrome and co-existing neurobehavioural disorders. J Intellect Disabil Res 2015;59:1130-1141.

16. Skogan AH, Zeiner P, Egeland J, Urnes AG, Reichborn-Kjennerud T, Aase H. Parent ratings of executive function in young preschool children with symptoms of attention-deficit/-hyperactivity disorder. Behav Brain Funct 2015;11:16.

17. Ezpeleta L, Granero R, de la Osa N, Penelo E, Domènech JM. Dimensions of oppositional defiant disorder in 3-year-old preschoolers. J Child Psychol Psychiatry 2012;53:1128-1138.

18. Marks DJ, Berwid OG, Santra A, Kera EC, Cyrulnik SE, Halperin JM. Neuropsychological correlates of ADHD symptoms in preschoolers. Neuropsychology 2005;19:446-455.

19. Gokler B, Oktem F, Çengel Kültür E. Attention Deficit Hyperactivity Disorder (ADHD) in Early Childhood. Unpublished Project Report no: 107S437. The Scientific and Technological Research Council of Turkey (TÜBITAK) ; 2009.

20. Kaufman J, Birmaher B, Brent D, Rao U, Flynn C, Moreci P, et al. Schedule for Affective Disorders and Schizophrenia for School-Age Children-Present and Lifetime Version (K-SADS-PL): initial reliability and validity data. J Am Acad Child Adolesc Psychiatry 1997;36:980-988.

21. Birmaher B, Ehmann M, Axelson DA, Goldstein BI, Monk K, Kalas C, et al. Schedule for affective disorders and schizophrenia for school-age children (K-SADS-PL) for the assessment of preschool children--a preliminary psychometric study. J Psychiatr Res 2009;43:680-686.

22. Gökler B, Ünal F, Pehlivantürk B, Kültür EÇ, Akdemir D, Taner Y. Reliability and Validity of Schedule for Affective Disorders and Schizophrenia for School Age Children-Present and Lifetime Version-Turkish Version (K-SADS-PL-T). Turk J Child Adolesc Ment Health 2004; $11 ; 109-116$

23. Conners CK. The Conners Rating Scales-Revised Manual. North Towanda, NY: Multi-Health Systems; 1997.

24. Kaner S, Büyüköztürk Ş, İşeri E. Psychometric properties of the Conners' Parent Rating Scale-Revised/Short Form (CPRS-R/S). International Developmental Neuropsychiatry Meetings III. İstanbul-Türkiye, 80-81; 2006.

25. Gioia GA, Isquith PK, Retzlaff PD, Espy KA. Confirmatory factor analysis of the Behavior Rating Inventory of Executive Function (BRIEF) in a clinical sample. Child Neuropsychol 2002;8:249-257.

26. Raid GH. Stanford-Binet Intelligence Scale (5th Ed). Rolling Meadows, IL: Riverside Publishing; 2003.

27. Cohen J. Statistical Power Analysis for the Behavioral Sciences (2nd Ed). Hillsdale, NJ: Lawrence Erlbaum Associates Inc; 1988.

28. Ezpeleta L, Granero R. Executive functions in preschoolers with ADHD, ODD, and comorbid ADHD-ODD: Evidence from ecological and performance-based measures. J Neuropsychol 2015;9:258-270.

29. Mahone EM. Measurement of attention and related functions in the preschool child. Ment Retard DevDisabil Res Rev 2005;11:216-225.

30. Kalff AC, Hendriksen JG, Kroes M, Vles JS, Steyaert J, Feron FJ, et al. Neurocognitive performance of 5- and 6-year-old children who met criteria for attention deficit/hyperactivity disorder at 18 months followup: results from a prospective population study. J Abnorm Child Psychol 2002;30: 589-598.

31. Skogan AH, Zeiner P, Egeland J, Rohrer-Baumgartner N, Urnes AG, Reichborn-Kjennerud T, et al. Inhibition and working memory in young preschool children with symptoms of ADHD and/or oppositional-defiant disorder. Child Neuropsychol 2014;20:607-624.

32. Sonuga-Barke EJ, Dalen L, Daley D, Remington B. Are planning, working memory, and inhibition associated with individual differences in preschool ADHD symptoms? Dev Neuropsychol 2002;21:255-272. 
33. Thorell LB, Wåhlstedt C. Executive functioning deficits in relation to symptoms of ADHD and/or ODD in preschool children. Infant Child Dev 2006;15:503-518.

34. Berwid OG, Curko Kera EA, Marks DJ, Santra A, Bender HA, Halperin JM. Sustained attention and response inhibition in young children at risk for Attention Deficit/Hyperactivity Disorder. J Child Psychol Psychiatry 2005;46:1219-1229.

35. Byrne JM, Bawden HN, DeWolfe NA, Beattie TL. Clinical assessment of psychopharmacological treatment of preschoolers with ADHD. J Clin Exp Neuropsychol 1998;20:613-627.

36. Harper GW, Ottinger DR. The performance of hyperactive and control preschoolers on a new computerized measure of visual vigilance: the Preschool Vigilance Task. J Child Psychol Psychiatry 1992;33:13651372.
37. Mahone EM, Pillion JP, Hoffman J, Hiemenz JR, Denckla MB. Construct validity of the auditory continuous performance test for preschoolers. Dev Neuropsychol 2005;27:11-33.

38. von Stauffenberg C, Campbell SB. Predicting the early developmental course of symptoms of attention deficit hyperactivity disorder. J Appl Dev Psychol 2007;28:536-552.

39. Barnard H, Rao R, Xu Y, Froehlich T, Epstein J, Lanphear BP, et al. Association of the Conners' Kiddie Continuous Performance Test (K-CPT) Performance and Parent-Report Measures of Behavior and Executive Functioning. J Atten Disord 2015 [Epub ahead of print].

40. Espy KA, Sheffield TD, Wiebe SA, Clark CA, Moehr MJ. Executive control and dimensions of problem behaviors in preschool children. J Child Psychol Psychiatry 2011;52:33-46. 